\title{
Association of early-onset constipation and diarrhea with patient outcomes in critically ill ventilated patients: a retrospective observational cohort study
}

Gen Aikawa ( $\sim$ gethoon@gmail.com )

University of Tsukuba

Akira Ouchi

Ibaraki Christian University

Hideaki Sakuramoto

Ibaraki Christian University

Tetsuya Hoshino

University of Tsukuba

Yuki Enomoto

University of Tsukuba

Nobutake Shimojo

University of Tsukuba

Yoshiaki Inoue

University of Tsukuba

\section{Research Article}

Keywords: diarrhea, constipation, patients, admission, critically, defecation

Posted Date: October 27th, 2021

DOl: https://doi.org/10.21203/rs.3.rs-966931/v1

License: (c) (i) This work is licensed under a Creative Commons Attribution 4.0 International License.

Read Full License 


\section{Abstract}

Constipation and diarrhea are both associated with poor outcomes in critically ill patients. Although constipation and diarrhea are closely related, few studies have examined them simultaneously. The purpose of this study was to describe patient defecation status after intensive care unit (ICU) admission and determine the association of early-onset constipation and diarrhea after ICU admission with outcomes for critically ill ventilated patients. Critically ill patients were retrospectively investigated and their defecation status was assessed during the first week after admission. The patients were divided into three groups: normal defecation, constipation, and diarrhea, and multiple comparison tests were performed. Additionally, multivariable analysis was performed for mortality and length of stay. Of the 85 critically ill ventilated patients, 47 (55\%) experienced constipation, and 12 (14\%) experienced diarrhea during the first week of ICU admission. Patients with normal defecation and diarrhea increased from the fourth and fifth day of ICU admission. Diarrhea was significantly associated with the length of ICU stay $(B=7.534,95 \%$ confidence interval: 0.116-14.951). Early-onset constipation and diarrhea were common in critically ill ventilated patients, and early-onset diarrhea was associated with the length of ICU stay. Prevention of constipation and diarrhea before the fifth day of ICU admission is essential.

\section{Introduction}

In recent years, various guidelines have recommended the early initiation of enteral nutrition (EN) for critically ill patients who require intensive care $(1-3)$. However, critically ill patients often develop gastrointestinal (GI) intolerances such as vomiting, regurgitation, abdominal distension, constipation, and diarrhea, and thus EN may not always be appropriate or properly implemented $(4,5)$.

GI motility in critically ill patients can be impaired by many factors, including ischemia, analgesics, vasoconstrictors, fluid management, and comorbidities such as diabetes (6). Between $34-58 \%$ of critically ill patients experience constipation as a result of GI dysmotility (7). In long-term ventilated patients, adverse symptoms associated with constipation include not only the typical symptoms such as abdominal distension, nausea, and vomiting, but also an increased incidence of acquired infections such as ventilator-associated pneumonia and bloodstream infections (8). A recent meta-analysis reported that constipation was associated with mechanical ventilation (MV) and intensive care unit (ICU) stay in critically ill patients (9).

Laxatives are commonly used in clinical practice to treat constipation in critically ill patients; however, the above-mentioned meta-analysis further reported that prophylactic laxatives caused significantly more diarrhea in the intervention group and had no effect on patient mortality (9). Diarrhea is a common symptom of intestinal inflammation and antibiotic administration in critically ill patients, causing dehydration, electrolyte abnormalities, and incontinence-associated dermatitis, increasing the burden on caregivers (10). It was also reported that diarrhea was associated with longer ICU and hospital stays and ICU mortality (11). 
Although constipation and diarrhea are closely related, as described above, many studies focus on either constipation or diarrhea, and there are few studies that have investigated constipation and diarrhea simultaneously. Therefore, the purpose of this study was to determine the defecation status during the first week after ICU admission and to clarify the association of constipation and diarrhea in the early stage of ICU admission with the outcome for critically ill patients.

\section{Results}

\section{Characteristics of patients}

During the recruitment period, 703 patients were admitted to the ICU, of whom, 148 were eligible. Of those, 63 patients were excluded and finally 85 patients were available for analysis (Supplementary Fig. S1 online). The patients were characterized as follows; 55 (65\%) were male, mean age was 67 (5677) years, BMI was 22.6 (20.1-26.5) kg/m², APACHE II score was 27 (22-30), and 38 (45\%) had cardiovascular disease.

\section{Enteral nutrition and the defecation status after ICU admission}

Figure 1 shows the percentage of EN and the progress of defecation during the first week after admission. EN was started in $41 \%$ of the patients on the second day of admission, rising to $86 \%$ by the fifth day. The percentage of patients with normal defecation increased on the fourth day, and the percentage of patients with diarrhea increased on the fifth day. Normal defecation occurred on day 3 (2-5) in the normal defecation group, and diarrhea occurred on day $6(2-6)$ in the diarrhea group.

\section{Comparison of normal defecation, constipation, and diarrhea}

The patients were divided into three groups: 26 (31\%) with normal defecation, 47 (55\%) with constipation, and 12 (14\%) with diarrhea. There was no significant difference between the three groups regarding background data (Table 1 ). In the medication data, there was a significant difference in propofol doses of 6.9 (1.3-13.1) mg/kg/day in the normal defecation group, $11.4(1.9-24.3) \mathrm{mg} / \mathrm{kg} / \mathrm{day}$ in the constipation group, and $1.0(0.1-6.2) \mathrm{mg} / \mathrm{kg} /$ day in the diarrhea group ( $p=0.015)$ (Table 2); pairwise comparison showed a significant difference between constipation and diarrhea groups $(p=0.013)$. There was no significant difference in EN, new-onset dialysis, MV duration, and infection data, but fluid overload was significantly different in $3(12 \%), 19(40 \%)$, and $3(25 \%)$ patients, respectively ( $p=0.032)$ (Table 3$)$, and pairwise comparison was significantly different between the normal defecation group and the constipation group $(p=0.030)$. There was a significant difference in ICU stay of $11(9-13), 13(9-21)$, and 20 (11-28) days, respectively ( $p=0.039)$, and pairwise comparison showed a significant difference between the normal defecation group and the diarrhea group $(p=0.045)$ (Fig. 2). There was no significant difference in mortality (Fig. 3).

\section{Association of constipation and diarrhea with patient outcomes}


In the multivariable analysis, while constipation was not associated with length of ICU stay ( $p=0.118)$, diarrhea, unlike constipation, was significantly associated with length of ICU stay (partial regression coefficient $[\mathrm{B}]=7.534,95 \% \mathrm{Cl}$ : 0.116-14.951, $p=0.047$ ) (Table 4). Neither constipation nor diarrhea was associated with mortality ( $p=0.327 ; p=0.327$, respectively) (Table 5).

\section{Discussion}

This study had three main findings. First, $55 \%$ of critically ill ventilated patients experienced constipation and $14 \%$ experienced diarrhea within the first week of ICU admission. Second, more patients had normal defecation from the fourth day, and diarrhea increased from the fifth day of ICU admission. Third, neither constipation nor diarrhea was associated with mortality, but diarrhea was associated with length of ICU stay.

In previous studies, $34-58 \%$ of patients had constipation, which was comparable with the result of this study $(7,8)$. The incidence of constipation varied among previous studies because of inconsistent definitions of constipation (9). Although many previous studies have used the definition of no defecation $\geq 3$ days, Prat and colleagues reported that patients who had no defecation $\geq 6$ days had more adverse outcomes than other patients (12). Therefore, in the current study, we confidently defined constipation as $\geq 6$ days without defecation from ICU admission.

The incidence of diarrhea also varies widely among many studies, from 10-78\% (9). In addition to the reasons for the different definitions as described above, this is also attributable to the method of data collection. The longer the number of days for which data are collected, the greater the chance that the patient will experience diarrhea, and thus the incidence could be higher. However, in our study, the effect was removed by conditioning the event to diarrhea occurrences during the first week of ICU admission. A previous study investigating GI symptoms during the first week of ICU admission reported that $22 \%$ of patients experienced diarrhea, indicating that diarrhea is common in ICU patients (13). However, because our study included only patients who used MV for more than 48 hours, there are no other studies with the same study population, which make our findings difficult to compare to those of past studies.

Similar to the previously-mentioned meta-analysis study (11), diarrhea was associated with length of ICU stay. The mechanism is not obvious, but diarrhea could cause impaired nutritional intake, electrolyte abnormalities, and dehydration, which may interfere with the critically ill patient's recovery. Taito and colleagues suggest that, because the causality between diarrhea and ICU length of stay is unknown and the reverse causality described above cannot be ruled out, further studies are needed to evaluate the association between early-onset diarrhea and ICU length of stay (11). Our study study is one of the few that have addressed this issue by defining the occurrence as within the first week of ICU admission.

The meta-analysis also reported that diarrhea was associated with mortality, which was different from the results of our study (11). However, as mentioned above, many studies do not limit the observation period. Therefore, while late-onset diarrhea may be relevant, from the results of our study we can at least say that early-onset diarrhea was not associated with prognosis. 
Constipation was not associated with patient outcomes, but over half of the critically ill patients in our study experienced constipation. Even though EN is started on the third day in many patients, having no defecation $\geq 6$ days is distressing for patients. Taken together, our findings suggest that prevention of both constipation and diarrhea is essential for critically ill patients. One method of constipation and diarrhea prevention could be a bowel management protocol; however it has been shown that the use of laxatives for constipated patients increases the risk of diarrhea occurrence (9). Hence, we propose the use of an intervention involving a gut immune protection bundle with the addition of symbiotics using a combination of dietary fiber and beneficial bacteria, all of which are known to be effective against diarrhea (14). Furthermore, this intervention may be more effective if it is introduced before the fifth day after ICU admission when the occurrence of diarrhea was observed to increase. If diarrhea does occur, it is also important to take prompt action against electrolyte abnormalities and dehydration. In particular, appropriate monitoring of laboratory values and early detection of dehydration as well as electrolyte supplementation and fluid management are required (15).

When investigating constipation or diarrhea typically one would divide them directly into two groups representing those with and without occurrence of the event; however, if we compare a group with constipation and a group without constipation, for example, patients with diarrhea may be included in the group without constipation. Previous studies have not excluded these patients and, as such, the study design might have distorted the results. We were able to eliminate this misclassification bias by dividing the participants into three groups: normal defecation, constipation, and diarrhea. Furthermore, this study is novel in that it describes the status of EN and defecation during the first week of ICU admission, which has not been done in previous studies (11). Especially, investigation on the timing of the commencement of diarrhea is particularly lacking in the literature, thus our study could be a valuable resource.

This study has several limitations. First, all data were collected retrospectively based on electronic medical records, thus, unrecorded data could be not retrieved. At the facility under observation, information on the administration of injectable drugs was available, but information on the administration of oral drugs was not incorporated into the electronic medical record. Consequently, the use of intestinal peristalsis promoting drugs, a risk factor for diarrhea, was unknown and could not be analyzed. In addition, the formulation and administration method of EN was based on the judgment of clinicians and as such was varied. To solve these problems, we plan to conduct a daily prospective observational study after the EN protocol is implemented. Second, our findings are limited by the small number of diarrhea cases; there were 12 patients with early onset of diarrhea during the one-year observation period. It would be necessary to observe at least four years of data to eliminate chance errors. Third, since this study was conducted at a single university hospital, these results may not be generalizable to community hospitals or other institutions. Therefore, to increase the generalizability of the findings the number of research facilities may need to be increased in future studies. Finally, because the recorded details of the nature and amount of defecation were dependent on the subjective evaluation of the nursing staff, they may have overestimated or underestimated the occurrence or severity of the diarrhea. We aimed to avoid this by using a semi-quantitative tool, but it might be better to introduce a more systematic instrument such as the Bristol stool form scale or King's stool chart (16-18). 


\section{Conclusions}

In critically ill ventilated patients, early-onset constipation and early-onset diarrhea occurred occasionally; however, only early-onset diarrhea was associated with the length of ICU stay. In ICU practice, early use of gut immune protection bundles with additional of symbiotics such as dietary fiber and beneficial bacteria, could be effective against diarrhea.

\section{Methods}

\section{Setting and patient selection}

This study was conducted at the ICU of the University of Tsukuba Hospital, Japan, which has 800 beds. Our ICU has surgical and emergency ICUs with 12 beds and admits 700-800 patients annually. There was no protocol for interventions for constipation or diarrhea, and GI peristalsis medications and enemas were administered at the discretion of doctors and nurses.

The participants were selected from all patients who were admitted to the ICU over a one-year period from April 2018 to March 2019. Inclusion criteria were patients aged over 18 years requiring MV $\geq 48$ hours, and in whom MV was induced between 24 hours before and 48 hours after ICU admission. Exclusion criteria were as follows: after GI surgery and/or colostomy, with GI diseases, bloody stool, readmission, withdrawal from aggressive treatment, and total length of ICU stay $<6$ days. The reason for the 6 -day cut off point was to match the definition of constipation as described below.

\section{Data collection}

This study design was a retrospective observational cohort study and patient data were collected from electronic medical records. Age, sex, comorbidities, diseases for ICU admission, Acute Physiology and Chronic Health Evaluation (APACHE) II scores for illness severity score, body mass index (BMI), defecation status, fluid intake and output, laboratory data, medication data, EN status, duration of ventilation, infection data, ICU and hospital outcomes were recorded. Fluid overload was defined as a $10 \%$ increase in body weight on the second day of admission compared to baseline (the first day of admission or before surgery) (19). When EN had been started within 48 hours of admission, the patient was considered as being on early EN (3).

Defecation was assessed by nurses using a semi-quantitative tool and was classified as follows: "large amount of liquid stool," "moderate amount of loose stool," "small amount of hard stool" and so on. Constipation was defined as " $\geq 6$ days without defecation" from ICU admission $(7,8,12)$. In accordance with previous studies (11) diarrhea was defined as "loose or liquid stools $\geq 3$ per day" occurring within the first week of ICU admission. According to the above definitions, the participants were divided into three groups (normal defecation, constipation, and diarrhea) based on their defecation status during the first week in the ICU. The primary endpoints were the association of constipation and diarrhea with patient outcomes (the length of stay and mortality). 


\section{Statistical analysis}

Descriptive data were expressed as numbers and percentages, and continuous data were expressed as median and interquartile range. For categorical variables, the $\chi$-square test or Fisher's exact test was used for comparisons between the three groups and for pairwise comparisons. For continuous variables, the Kruskal-Wallis test was used for comparisons between the three groups, and the Mann-Whitney $U$ test was used for pairwise comparisons. Bonferroni adjustment was used for each pairwise comparison. The association of constipation and diarrhea with patient outcomes were examined using linear and logistic regression analysis. When analyzing constipation, diarrhea was removed to compare constipation with normal defecation, and when analyzing diarrhea, constipation was removed as well. The results are reported as partial regression coefficients ( $95 \%$ confidence interval [CI]) for mortality and odds ratios (95\% $\mathrm{Cl}$ ) for the length of stay. Adjustments were made for the following additional covariates that were chosen a priori: APACHE II score, fluid overload $(6,20)$. Differences with $p$ values of $<0.05$ indicated statistical significance. All analyses were conducted with IBM SPSS Statistics 27 (IBM Corp., Armonk, N.Y., USA).

\section{Declarations}

\section{Ethics approval and consent to participate}

This study was approved by the Institutional Review Board of the University of Tsukuba (Approval \#R01163). Because this study was retrospective in design and used anonymized patient data, informed consent from each patient was not required. Instead, an opt-out protocol was implemented and patients were given the opportunity to refuse consent to participate. All procedures were approved according to the regulations of the University of Tsukuba that equal or exceed the standards set by the Declaration of Helsinki.

\section{Availability of data and materials}

All data generated or analyzed during this study are included in Supplementary Data S2 online.

\section{Author contributions}

GA, AO and HS: Study design. GA and AO: Data collection. GA, AO and HS: Statistical analysis. GA: Manuscript documentation. AO, HS, TH, YE, NS and YI: Revision. All authors read and approved the final manuscript.

\section{Additional information}

\section{Competing interests}

The authors declare that they have no competing interests 


\section{Funding}

The education and research expenses for the Department of Emergency and Critical Care Medicine of University of Tsukuba were used for the English proofreading cost of the manuscript.

\section{References}

1. Singer, P. et al. ESPEN guideline on clinical nutrition in the intensive care unit. Clin. Nutr, 38, 48-79 https://doi.org/10.1016/j.clnu.2018.08.037 (2019).

2. McClave, S. A. et al. Guidelines for the Provision and Assessment of Nutrition Support Therapy in the Adult Critically III Patient: Society of Critical Care Medicine (SCCM) and American Society for Parenteral and Enteral Nutrition (A.S.P.E.N.). JPEN J. Parenter. Enteral Nutr, 40, 159-211 https://doi.org/10.1177/0148607115621863 (2016).

3. Reintam Blaser, A. et al. Early enteral nutrition in critically ill patients: ESICM clinical practice guidelines. Intensive Care Med, 43, 380-398 https://doi.org/10.1007/s00134-016-4665-0 (2017).

4. Montejo, J. C. Enteral nutrition-related gastrointestinal complications in critically ill patients: A multicenter study. Crit. Care Med, 27, 1447-1453 https://doi.org/10.1097/00003246-19990800000006 (1999).

5. Reintam Blaser, A. et al. Gastrointestinal dysfunction in the critically ill: a systematic scoping review and research agenda proposed by the Section of Metabolism, Endocrinology and Nutrition of the European Society of Intensive Care Medicine. Crit. Care, 24, 224 https://doi.org/10.1186/s13054-02002889-4 (2020).

6. Fruhwald, S. \& Kainz, J. Effect of ICU interventions on gastrointestinal motility. Curr. Opin. Crit. Care, 16, 159-164 https://doi.org/10.1097/MCC.0b013e3283356679 (2010).

7. Fukuda, S. et al. Risk factors for late defecation and its association with the outcomes of critically ill patients: a retrospective observational study. J Intensive Care, 4, 33 https://doi.org/10.1186/s40560016-0156-1 (2016).

8. Gacouin, A. et al. Constipation in long-term ventilated patients: associated factors and impact on intensive care unit outcomes. Crit. Care Med, 38, 1933-1938 https://doi.org/10.1097/CCM.0b013e3181eb9236 (2010).

9. Hay, T. et al. Constipation, diarrhea, and prophylactic laxative bowel regimens in the critically ill: A systematic review and meta-analysis. J. Crit. Care, 52, 242-250 https://doi.org/10.1016/j.jcrc.2019.01.004 (2019).

10. de Brito-Ashurst, I. \& Preiser, J. C. Diarrhea in Critically III Patients: The Role of Enteral Feeding. JPEN J. Parenter. Enteral Nutr, 40, 913-923 https://doi.org/10.1177/0148607116651758 (2016).

11. Taito, S. et al. Diarrhea and patient outcomes in the intensive care unit: Systematic review and metaanalysis. J. Crit. Care, 53, 142-148 https://doi.org/10.1016/j.jcrc.2019.06.014 (2019).

12. Prat, D. et al. Constipation in critical care patients: both timing and duration matter. Eur. J. Gastroenterol. Hepatol, 30, 1003-1008 https://doi.org/10.1097/MEG.0000000000001165 (2018). 
13. Reintam Blaser, A. et al. Gastrointestinal symptoms during the first week of intensive care are associated with poor outcome: a prospective multicentre study. Intensive Care Med, 39, 899-909 https://doi.org/10.1007/s00134-013-2831-1 (2013).

14. Shimizu, K. et al. Synbiotics modulate gut microbiota and reduce enteritis and ventilator-associated pneumonia in patients with sepsis: a randomized controlled trial. Crit. Care, 22, 239 https://doi.org/10.1186/s13054-018-2167-x (2018).

15. Reintam Blaser, A., Deane, A. M. \& Fruhwald, S. Diarrhoea in the critically ill. Curr. Opin. Crit. Care, 21, 142-153 https://doi.org/10.1097/MCC.0000000000000188 (2015).

16. O'Donnenll, L. J. D., Virjee, J. \& Heaton, K. W. Detection of pseudodiarrhoea by simple clinical assessment of intestinal transit rate. $B M J, 300,439-440 \mathrm{https} / / /$ doi.org/10.1136/bmj.300.6722.439 (1990).

17. Blake, M. R., Raker, J. M. \& Whelan, K. Validity and reliability of the Bristol Stool Form Scale in healthy adults and patients with diarrhoea-predominant irritable bowel syndrome. Aliment. Pharmacol. Ther, 44, 693-703 https://doi.org/10.1111/apt.13746 (2016).

18. Whelan, K., Judd, P. A. \& Taylor, M. A. Assessment of fecal output in patients receiving enteral tube feeding: validation of a novel chart. Eur. J. Clin. Nutr, 58, 1030-1037 https://doi.org/10.1038/sj.ejcn.1601927 (2004).

19. Chapalain, X. et al. Association between fluid overload and SOFA score kinetics in septic shock patients: a retrospective multicenter study. J Intensive Care, 7, 42 https://doi.org/10.1186/s40560019-0394-0 (2019).

20. Hansen, B. Fluid Overload. Front Vet Sci, 8, 668688 https://doi.org/10.3389/fvets.2021.668688 (2021).

\section{Tables}


Table 1. Comparison of patient background data among the normal defecation, constipation, and diarrhea groups

\begin{tabular}{|c|c|c|c|c|c|c|c|}
\hline \multirow{2}{*}{$\begin{array}{l}\text { Variables } \\
\text { Age, median (IQR) }\end{array}$} & \multicolumn{2}{|c|}{ Normal $(n=26)$} & \multicolumn{2}{|c|}{$\begin{array}{l}\text { Constipation } \\
(n=47)\end{array}$} & \multicolumn{3}{|c|}{$\begin{array}{l}\text { Diarrhea } \\
(n=12)\end{array}$} \\
\hline & 62 & $(52-79)$ & 67 & $(57-76)$ & 73 & $(58-77)$ & 0.658 \\
\hline Male, n (\%) & 15 & $(57)$ & 35 & (74) & 5 & $(42)$ & 0.070 \\
\hline BMI, mean (SD) & 22.0 & $\begin{array}{l}(20.1- \\
26.1)\end{array}$ & 23.0 & $\begin{array}{l}(20.2- \\
27.1)\end{array}$ & 21.4 & $\begin{array}{l}(18.7- \\
23.5)\end{array}$ & 0.319 \\
\hline APACHE, median (IQR) & 26 & $(21-34)$ & 27 & $(21-30)$ & 30 & $(26-31)$ & 0.214 \\
\hline \multicolumn{8}{|c|}{ Disease for ICU admission, $\mathrm{n}(\%)$} \\
\hline Cardiovascular & 8 & (31) & 26 & $(55)$ & 4 & (33) & 0.090 \\
\hline Respiratory & 6 & (23) & 6 & (13) & 5 & $(42)$ & 0.074 \\
\hline Cardiopulmonary arrest $^{\mathrm{a}}$ & 5 & (19) & 6 & (13) & 2 & $(17)$ & 0.699 \\
\hline Neurology ${ }^{a}$ & 3 & $(12)$ & 8 & $(17)$ & 0 & (0) & 0.398 \\
\hline Sepsis ${ }^{a}$ & 2 & (8) & 1 & (2) & 0 & (0) & 0.551 \\
\hline Burn $^{a}$ & 2 & (8) & 0 & (0) & 0 & $(0)$ & 0.197 \\
\hline Necrotizing fasciitis ${ }^{a}$ & 0 & $(0)$ & 0 & (0) & 1 & (8) & 0.141 \\
\hline \multicolumn{8}{|l|}{ Comorbidity, n (\%) } \\
\hline Hypertension & 6 & (23) & 17 & (36) & 2 & (17) & 0.290 \\
\hline Diabetes & 9 & (35) & 8 & (17) & 2 & (17) & 0.197 \\
\hline Chronic kidney disease & 7 & $(27)$ & 8 & (17) & 5 & $(42)$ & 0.177 \\
\hline Cancer $^{\mathrm{a}}$ & 5 & (19) & 7 & (15) & 4 & (33) & 0.333 \\
\hline Autoimmune disease ${ }^{a}$ & 1 & $(4)$ & 3 & $(6)$ & 1 & (8) & 0.839 \\
\hline \multicolumn{8}{|c|}{ Laboratory data, AUC, median (IQR) ${ }^{\mathrm{b}}$} \\
\hline White blood cell & 26.0 & $\begin{array}{l}\left(22.2^{-}\right. \\
31.2)\end{array}$ & 21.2 & $\begin{array}{l}(16.4- \\
30.9)\end{array}$ & 22.3 & $\begin{array}{l}\left(19.2^{-}\right. \\
28.4)\end{array}$ & 0.175 \\
\hline C-reactive protein & 24.18 & $\begin{array}{l}(20.58- \\
33.50)\end{array}$ & 31.82 & $\begin{array}{l}(21.42- \\
39.61)\end{array}$ & 26.77 & $\begin{array}{l}(15.59- \\
31.80)\end{array}$ & 0.170 \\
\hline Albumin & 5.0 & $(4.2-5.8)$ & 5.0 & $(4.2-5.5)$ & 4.3 & $(3.6-5.1)$ & 0.200 \\
\hline Creatinine & 2.15 & $\begin{array}{l}(1.25- \\
4.50)\end{array}$ & 2.12 & $\begin{array}{l}(1.59- \\
3.91)\end{array}$ & 4.00 & $\begin{array}{l}(2.19- \\
8.63)\end{array}$ & 0.293 \\
\hline
\end{tabular}


Estimated glomerular filtration rate

Total bilirubin
113.5

(46.6-

192.2)

1.5
$(1.2-2.5) \quad 2.3$

102.0

2.3
(57.4156.2)

59.5

(1.2-4.6)
(26.5-

128.5)

2.9

(0.813.9)

0.139

$\mathrm{BMI}$, body mass index; IQR, interquartile range; APACHE, Acute Physiology and Chronic Health Evaluation; ICU, intensive care unit; AUC, area under the curve.

${ }^{a}$ Fisher exact test was performed. All other categorical variables were subjected to the $\chi^{2}$ test. ${ }^{b}$ The AUC of the laboratory data was calculated from the values at the time of admission, peak, and one week later.

Table 2. Comparison of medication data between the normal defecation, constipation, and diarrhea groups

Variables

Fentanyl, $\mu \mathrm{g} / \mathrm{kg} /$ day, median (IQR)

Propofol, mg/kg/day, median (IQR)

Muscle relaxant agent use, $\mathrm{n}(\%)$

Vasoactive agent use, $\mathrm{n}(\%)^{\mathrm{a}}$

Multiple antibiotics use, $\mathrm{n}(\%)$

${ }^{a}$ Fisher exact test was performed. All other categorical variables were subjected to the $\chi^{2}$ test.

Normal $(\mathrm{n}=26)$

14.4

6.9

6

21

11

(42)

(23)


Table 3. Comparison of intensive care status between the normal defecation, constipation, and diarrhea groups

\begin{tabular}{|c|c|c|c|c|c|c|c|}
\hline Variables & Norm & $(n=26)$ & $\begin{array}{l}\text { Con } \\
(n=2\end{array}$ & ipation & $\begin{array}{l}\text { Diar } \\
(n=1\end{array}$ & & lue \\
\hline Early enteral nutrition, n (\%) & 17 & $(65)$ & 26 & (55) & 8 & (67) & 0.617 \\
\hline $\begin{array}{l}\text { Enteral nutrition free days, } \\
\text { median (IQR) }\end{array}$ & 2.0 & $\begin{array}{l}(1.0- \\
3.0)\end{array}$ & 2.0 & $(1.0-4.0)$ & 1.5 & $(1.0-3.8)$ & 0.311 \\
\hline Fluid overload, n (\%) & 3 & (12) & 19 & (40) & 3 & (25) & 0.032 \\
\hline New-onset dialysis ${ }^{\mathrm{a}}, \mathrm{n}(\%)$ & 3 & (12) & 6 & (13) & 3 & (25) & 0.511 \\
\hline $\begin{array}{l}\text { Mechanical ventilator days, } \\
\text { median (IQR) }\end{array}$ & 10.5 & $\begin{array}{l}(6.0- \\
20.5)\end{array}$ & 11 & $(6-17)$ & 20 & $\begin{array}{l}(11.3- \\
50.5)\end{array}$ & 0.074 \\
\hline $\begin{array}{l}\text { Ventilator-associated } \\
\text { pneumonia }{ }^{a}, \mathrm{n}(\%)\end{array}$ & 4 & (15) & 9 & (19) & 0 & (0) & 0.283 \\
\hline Bloodstream infection ${ }^{\mathrm{a}}, \mathrm{n}(\%)$ & 3 & (12) & 7 & (15) & 1 & (8) & 1.000 \\
\hline
\end{tabular}

Table 4. Multivariable analysis of factors associated with the length of ICU stay

$\begin{array}{llll}\text { Variables } & \text { B } & 95 \% \text { confidence interval } & p \text { value }\end{array}$

\section{Model 1: Constipation and normal defecation}

$\begin{array}{llll}\text { Constipation } & 6.082 & (-1.591-13.755) & 0.118\end{array}$

$\begin{array}{llll}\text { APACHE II score } & 0.313 & (-0.170-0.796) & 0.201\end{array}$

$\begin{array}{llll}\text { Fluid over } & -4.660 & (-12.556-3.235) & 0.243\end{array}$

$\begin{array}{lll}\text { Propofol dosage } & 0.230 & (-0.041-0.501)\end{array}$

\section{Model 2: Diarrhea and normal defecation}

Diarrhea

APACHE II score

Fluid over

Propofol dosage

Linear regression analysis was performed with the length of ICU stay as the dependent variable. B is the partial regression coefficient.

ICU, intensive care unit; APACHE, Acute Physiology and Chronic Health Evaluation.
0.047

0.666

0.041

0.733 
Table 5. Multivariable analysis of factors associated with 28-day mortality

Variables OR $\quad 95 \%$ confidence interval $\quad p$ value

Model 1: Constipation and normal defecation

\begin{tabular}{llll} 
Constipation & 2.402 & $(0.417-13.842)$ & 0.327 \\
\hline APACHE II score & 1.019 & $(0.912-1.138)$ & 0.741 \\
\hline Fluid over & 1.966 & $(0.195-19.809)$ & 0.566 \\
\hline Propofol dosage & 1.015 & $(0.956-1.078)$ & 0.624
\end{tabular}

Model 2: Diarrhea and normal defecation

\begin{tabular}{llll} 
Diarrhea & 2.600 & $(0.385-17.574)$ & 0.327 \\
\hline APACHE II score & 1.016 & $(0.889-1.162)$ & 0.812 \\
\hline Fluid over & 0.605 & $(0.045-8.170)$ & 0.705 \\
\hline Propofol dosage & 1.052 & $(0.969-1.142)$ & 0.230
\end{tabular}

Logistic regression analysis was performed with 28-day mortality as the dependent variable. OR, odds ratio; APACHE, Acute Physiology and Chronic Health Evaluation.

\section{Figures}




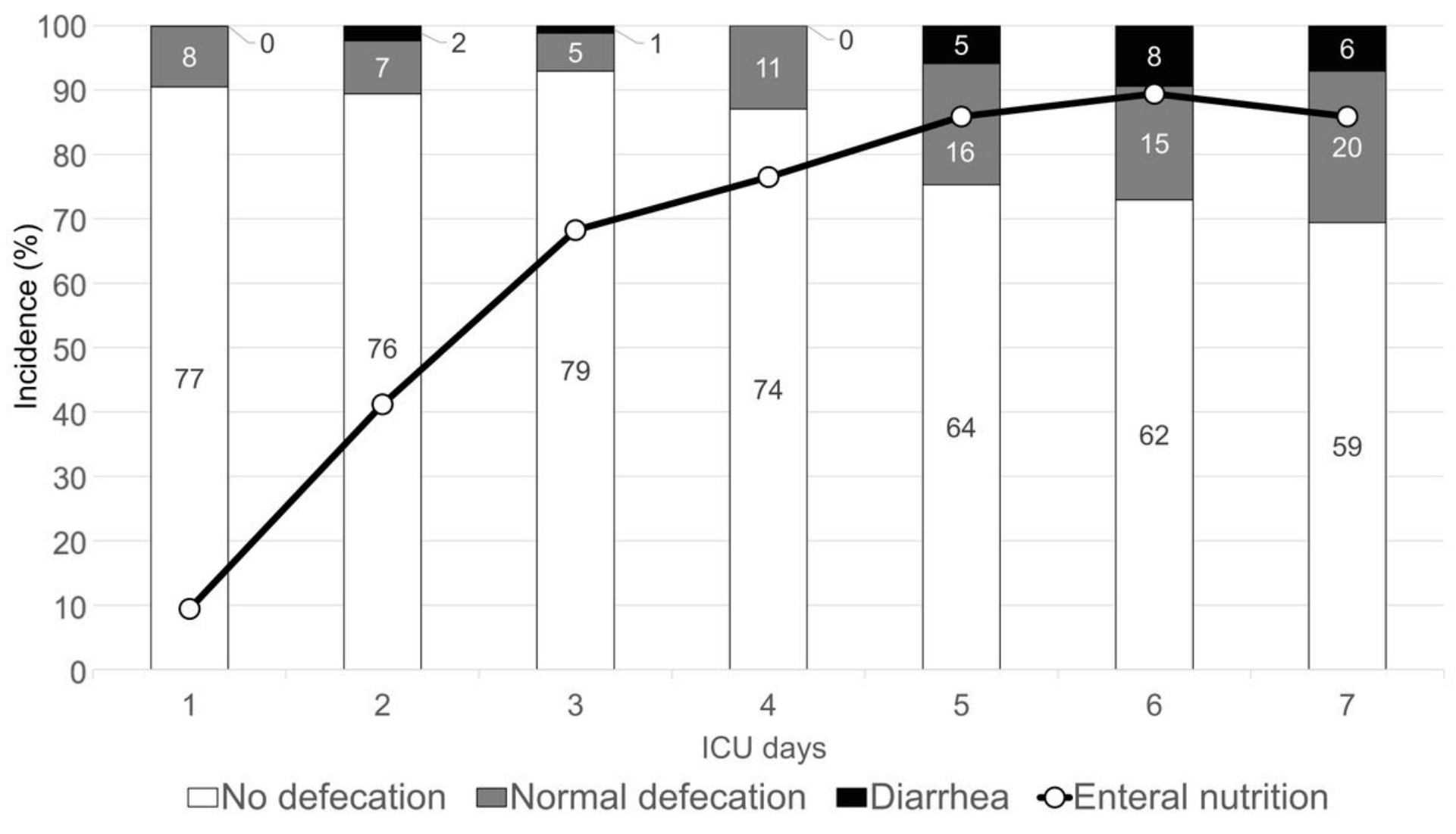

\section{Figure 1}

Temporal trends in enteral nutrition (EN) and defecation status after ICU admission. Daily EN and defecation status for the first week after ICU admission is shown as a percentage. Line graphs indicate EN, white bars indicate no defecation, gray bars indicate normal defecation, and black bars indicate diarrhea. EN was initiated in nearly half of the patients on day 2 after ICU admission. The proportion of patients gradually increased, and by day 5, most patients were receiving EN. The Proportion of patients with normal defecation increased from day 4 , and the number of patients with diarrhea increased from day 5. EN, enteral nutrition; ICU, intensive care unit. 

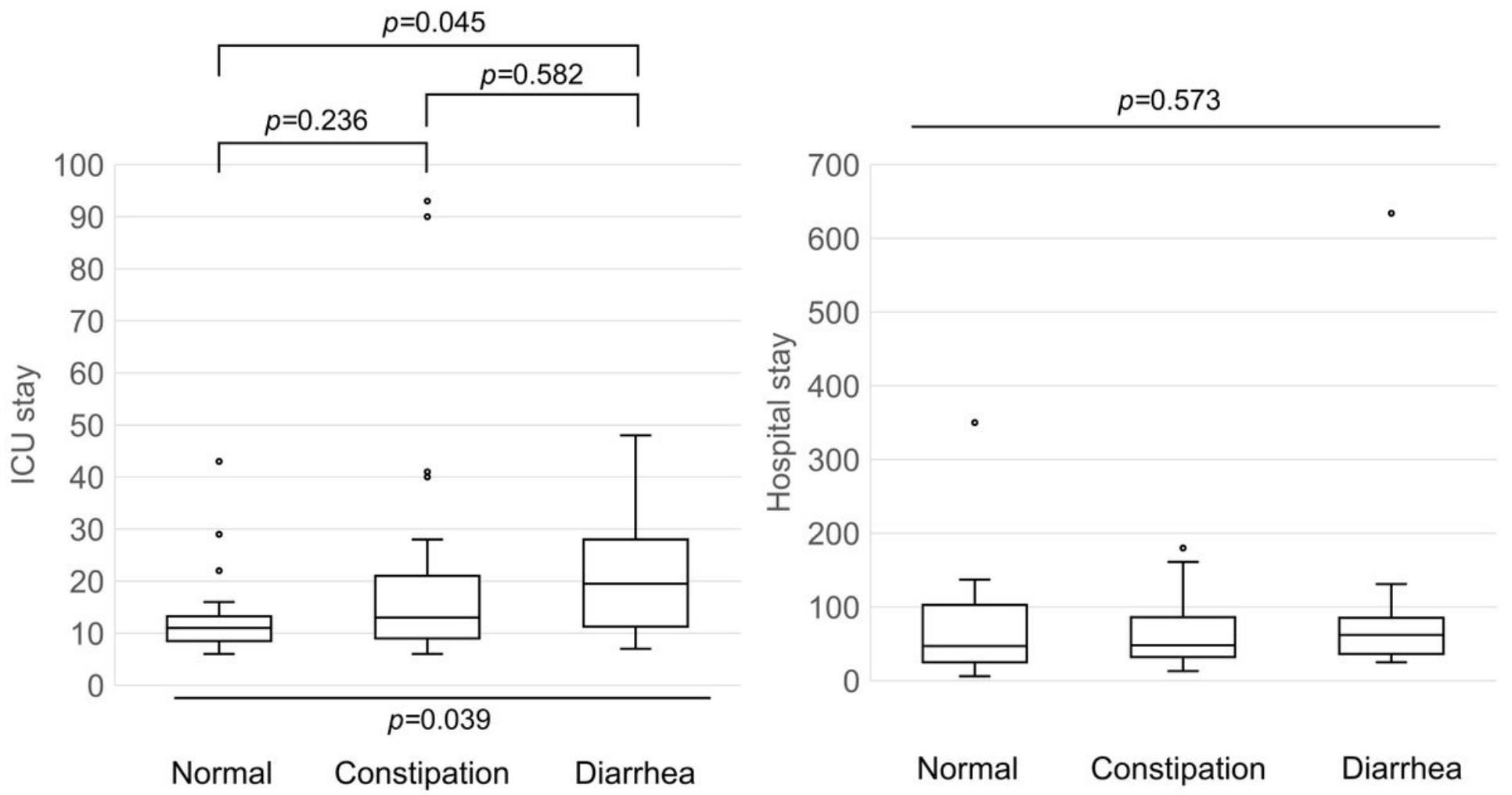

\section{Figure 2}

Comparison of length of stay in normal defecation, constipation, and diarrhea groups. The length of stay is represented as a box-and-whisker plot for the three groups: normal defecation, constipation, diarrhea. The left-hand graph is for length of ICU stay and that on the right is for length of hospital stay. The Kruskal-Wallis test was used for three-group comparison, followed by Mann-Whitney U test for pairwise comparisons. Bonferroni adjustment was used for each pairwise comparison. ICU, intensive care unit. 
90

80

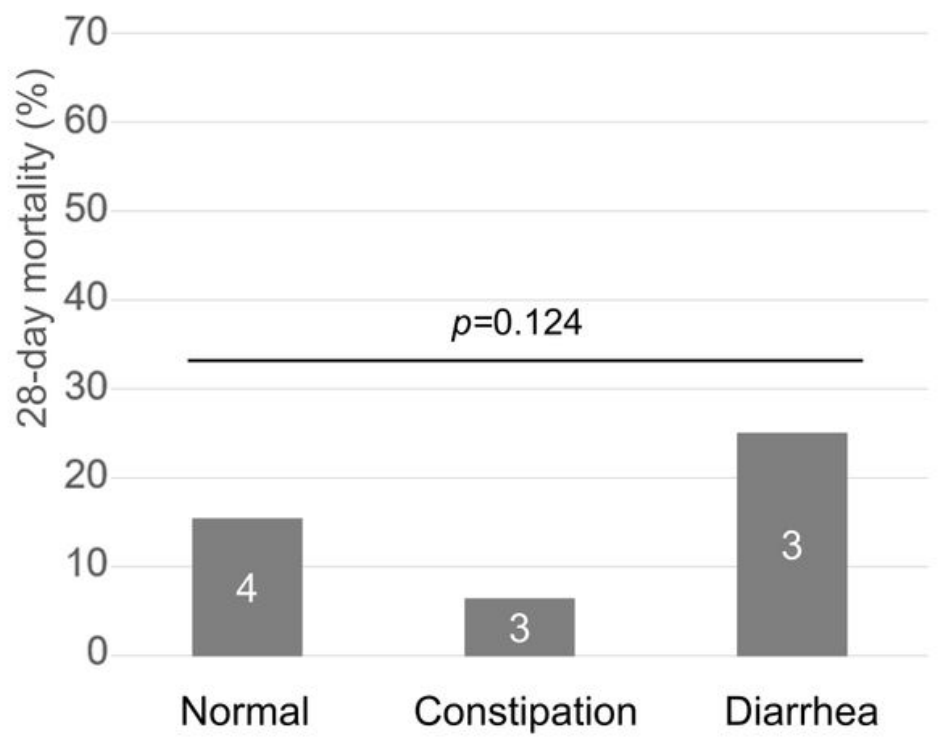

90

80

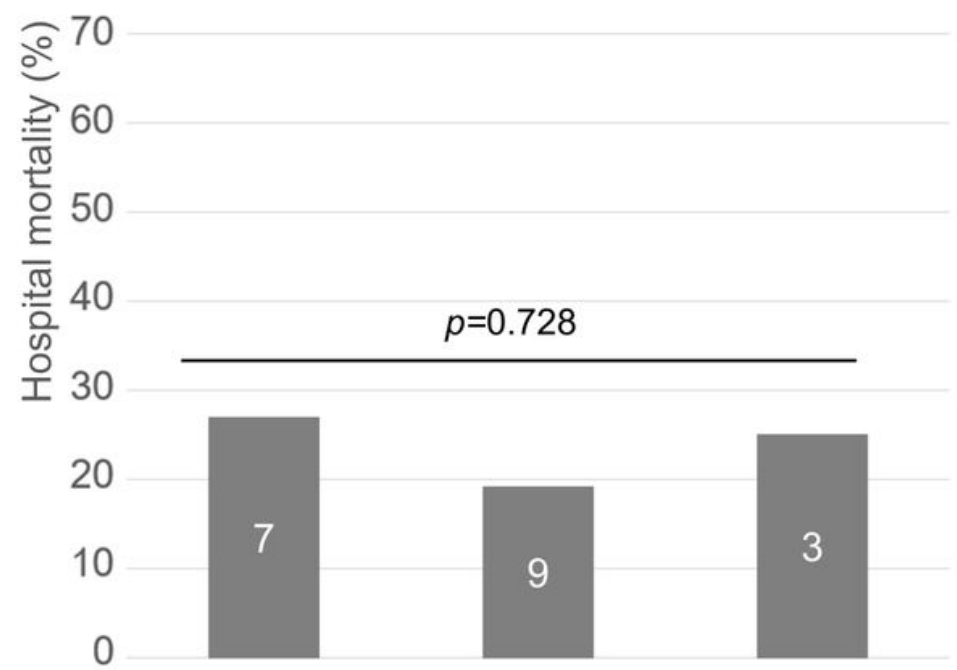

Normal Constipation
Diarrhea

\section{Figure 3}

Comparison of mortality among the normal defecation, constipation, and diarrhea groups. The mortality rate is represented in a $100 \%$ bar graph for three groups: normal defecation, constipation, and diarrhea. The left-hand graph represents 28-day mortality rate, and the right-hand shows the hospital mortality rate. The $\chi$-square test was used for the three-group comparison.

\section{Supplementary Files}

This is a list of supplementary files associated with this preprint. Click to download.

- SupplementaryFigureS1.docx

- SupplementaryDataS2.xIsx 